\title{
INVERTEBRADOS ASSOCIADOS A DETRITOS DE MACRÓFITAS AQUÁTICAS EM ÁREA ÚMIDA SUBTROPICAL DO SUL DO BRASIL
}

Invertebrates associated with aquatic macrophyte detrites in subtropical wet area of southern Brazil

\author{
Edélti Faria Albertoni ${ }^{1}$; Thais de Azevedo Carneiro² ${ }^{2}$ Cleber Palma-Silva ${ }^{1}$
}

\footnotetext{
${ }^{1}$ Programa de Pós-Graduação em Biologia de Ambientes Aquáticos Continentais, Universidade Federal do Rio Grande - FURG, Instituto de Ciências Biológicas, Laboratório de Limnologia. E-mail: efalbertoni@gmail.com

${ }^{2}$ Laboratório de Limnologia, Universidade Federal do Rio Grande - FURG, Instituto de Ciências Biológicas, Laboratório de Limnologia.
}

Data do recebimento: 05/11/2019 - Data do aceite: 06/12/2019

RESUMO: Macrófitas aquáticas são abundantes em ecossistemas rasos da Planície Costeira do Rio Grande do Sul. A velocidade de decomposição dessas plantas é regulada pela qualidade nutricional do detrito, pela presença de compostos inibidores de fitófagos e presença de invertebrados fragmentadores. Em sistemas subtropicais e tropicais, é relatada a virtual ausência de fragmentadores participando desse processo. Este estudo teve como objetivo principal avaliar a decomposição de três espécies de macrófitas aquáticas em uma área de banhado no Sul do Brasil. Foram medidos concentração de carbono e nitrogênio, teores de polifenóis e a comunidade de invertebrados, classificando-os em grupos tróficos funcionais. Os resultados demonstraram que a planta com maior concentração de nitrogênio e menor teor de polifenóis teve a maior velocidade de decomposição. A comunidade de invertebrados associados teve baixa presença de fragmentadores e maior abundância de raspadores. Sugerimos que o papel de fragmentação de detritos por invertebrados em áreas úmidas subtropicais pode estar sendo subestimado. Provavelmente, o detrito não está sendo utilizado como recurso alimentar, mas os mecanismos de comportamento alimentar de organismos raspadores auxiliam na quebra da matéria orgânica nesses sistemas, incrementando a velocidade de degradação. Palavras-chave: Pistia stratiotes. Salvinia herzogii. Eichhornia crassipes. Áreas Úmidas Subtropicais. Degradação Foliar. 


\begin{abstract}
Aquatic macrophytes are abundant in shallow ecosystems of the Rio Grande do Sul Coastal Plain. The rate of degradation of these plants is regulated by the nutritional quality of the debris, by the presence of phytophagous inhibitor compounds and by the presence of shredders invertebrates. It is reported the virtual absence of shredders participating in this process in subtropical and tropical systems. This study aimed to evaluate the decomposition of three aquatic macrophyte species in a wetland area in southern Brazil. Carbon and nitrogen concentration, polyphenol contents and the invertebrate community were measured, classifying them into functional trophic groups. The results showed that the plant with the highest nitrogen concentration and lowest polyphenol content had the highest decomposition rate. The associated invertebrate community had low presence of shredders and higher abundance of scrapers. It is suggested that the role of fragmentation of invertebrate debris in subtropical wetlands may be underestimated. Probably, waste is not being used as a food resource, but the mechanisms of feeding behavior of scraping organisms help the breakdown of organic matter in these systems, increasing the rate of degradation.
\end{abstract}

Keywords: Pistia stratiotes. Salvinia herzogii. Eichhornia crassipes. Subtropical Wetlands. Leaf Degradation.

\section{Introdução}

A decomposição de macrófitas aquáticas regula, em grande parte, a ciclagem de nutrientes em ecossistemas continentais rasos (POI DE NEIFF; NEIFF; CASCO, 2009). Essas plantas acumulam uma série de nutrientes do sedimento e da água circundante, que são liberados no ecossistema após a morte e início do processo de decomposição (ASAEDA; TRUNG; MANATUNGE, 2000). Em ecossistemas lênticos, como lagos e terras úmidas, o processo de decomposição ocorre junto ao sedimento, com fonte da matéria orgânica principalmente autóctone (ABELHO, 2001).

Ao longo do processo de decomposição ocorre a transformação da matéria orgânica particulada grossa (MOPG) em matéria orgânica particulada fina (MOPF) (WEBSTER; BENFIELD, 1986). As diferentes fases de lixiviação (perda inicial de compostos solúveis), condicionamento (colonização por microrganismos, principalmente fungos e bactérias, iniciando a quebra de moléculas orgânicas) e fragmentação (mecânica, por abrasão física, e biológica, pela ação principalmente de invertebrados, facilitada pelo condicionamento microbiano) são sobrepostas durante este processo (WEBSTER; BENFIELD, 1986; GESSNER; CHAUVET; DOBSON, 1999; GONÇALVES et al., 2014). Muitos fatores influenciam o processo de decomposição dos detritos em ambientes aquáticos, entre eles o teor nutricional, principalmente altas concentrações de nitrogênio (HEPP; DELANORA; TREVISAN, 2009), que favorece a colonização por invertebrados fragmentadores (BIASI et al., 2013). Outro fator que afeta o processo de decomposição é a presença de compostos químicos (como polifenóis e taninos), os quais inibem a ação 
tanto de microrganismos (fungos e bactérias) quanto dos invertebrados (GONÇALVES et al., 2014). A presença de invertebrados detritívoros é favorecida em detritos foliares com alto teor de nitrogênio e baixos teores de polifenóis (TONELLO et al., 2014).

A estrutura da comunidade de invertebrados associados aos detritos foliares é influenciada pela combinação das características estruturais (e.g. dureza) e químicas dos detritos foliares (e.g. concentração de nitrogênio e polifenóis), que aumentam ou diminuem sua palatabilidade e, consequentemente, facilitam ou dificultam a degradação (GRAÇA, 2001; GONÇALVES et al., 2014; TONELLO et al., 2014). Uma das formas para avaliar o papel da comunidade de invertebrados aquáticos na degradação foliar é avaliar seus hábitos alimentares, baseando-se nos mecanismos morfocomportamentais de aquisição e consumo de alimentos (TELÖKEN; ALBERTONI; PALMA-SILVA, 2011). Essa análise permite classificar os invertebrados em grupos tróficos funcionais (GTF), como coletores-filtradores, coletores-catadores, predadores, raspadores e fragmentadores (CUMMINS; MERRITT; ANDRADE, 2015). Várias pesquisas (DUDGEON; WU, 1999; TELÖKEN et al., 2014; CARVALHO et al., 2015; ALBERTONI et al., 2018) relatam a baixa densidade de invertebrados fragmentadores em sistemas lênticos tropicais e subtropicais, ressaltando que a comunidade associada ao detrito o utiliza primariamente como habitat, e não como recurso alimentar, não atuando diretamente na fragmentação da MOPG.

Os ecossistemas rasos da Planície Costeira Sul do Brasil são caracterizados pela presença de macrófitas aquáticas em grande densidade e diversidade (ROLON; MALTCHICK, 2006), resultando em altos níveis de produtividade primária (TRINDADE et al., 2010; PALMA-SILVA et al., 2012,) e proporcionando abrigo para uma grande diversidade de invertebrados aquáticos (ALBERTONI;
PRELLVITZ; PALMA-SILVA, 2007). Assim, o presente trabalho teve como principal objetivo avaliar o processo de decomposição e a colonização de invertebrados aquáticos em três macrófitas aquáticas abundantes em terras úmidas do Sul do Brasil. Nossas hipóteses foram que i: plantas com maior teor de nitrogênio e menor teor de polifenóis teriam decomposição mais rápida; ii: a colonização de invertebrados apresentaria maior densidade em plantas com maior tempo de decomposição, e iii: todos os detritos apresentariam baixa densidade de invertebrados fragmentadores.

\section{Material e Métodos}

\section{Área de estudo}

Realizamos o experimento de decomposição foliar de três macrófitas aquáticas - Salvinia herzogii de la Sota, Eichhornia crassipes Mart. (Solms) e Pistia stratiotes L. - abundantes na região da Planície Costeira Sul do Brasil, (ROLON; MALTCHICK, 2006) em uma área úmida localizada ao sul do município de Rio Grande, estado do Rio Grande do Sul, Brasil (32 $03^{\prime}$ SL 52 $\left.01^{\prime}\right)$. As características do local são semelhantes às das áreas úmidas comuns na Planície Costeira Sul, com baixas profundidades e grandes extensões de campos alagados, colonizados por macrófitas aquáticas (PALMA-SILVA et al., 2012).

\section{Caracterização Limnológica da Área Úmida}

O experimento de decomposição teve duração de 60 dias, entre outubro e dezembro de 2016. Para caracterizar limnologicamente o local de estudo, em cada tempo amostral, determinamos $\mathrm{pH}$, condutividade elétrica, oxigênio dissolvido, turbidez e temperatura da água, com auxílio de uma sonda multipa- 
râmetro HORIBA ${ }^{\circledR}$. A profundidade (medida com régua graduada $\mathrm{em} \mathrm{cm}$ ) no local variou entre 120 e $60 \mathrm{~cm}$. Durante o período estudado, ocorreu pouca variação na maioria das variáveis limnológicas. A temperatura variou entre 16,7 e $20,8^{\circ} \mathrm{C}$, o $\mathrm{pH}$ manteve-se levemente ácido, oscilando entre 4,52 e 7,1. Os valores de oxigênio mantiveram-se entre 6,18 e $11,5 \mathrm{mg} \cdot \mathrm{L}^{-1}$. A condutividade elétrica oscilou entre 0,09 e $0,20 \mathrm{mS} . \mathrm{cm}^{-1}$, e a turbidez entre 42 e 265 NTU.

\section{Procedimentos de Campo}

Coletamos as macrófitas S. herzogii, E. crassipes e $P$. stratiotes e as secamos ao ar livre em temperatura ambiente $\left(c a .25^{\circ} \mathrm{C}\right) \mathrm{du}-$ rante duas semanas. Após este procedimento, foram montadas 60 litter bags de tamanho $20 \mathrm{~cm} \times 30 \mathrm{~cm}$ e aberturas de malha de 0,1 $\mathrm{cm}^{2}$ na face em contato com o sedimento e $1,0 \mathrm{~cm}^{2}$ na face oposta, permitindo o acesso aos invertebrados colonizadores. Foram confeccionadas 60 bolsas - 20 para cada planta, contendo 10,0 $\pm 3,0$ gramas de material foliar de cada macrófita -, sendo identificadas com etiquetas de plástico. As bolsas foram colocadas junto ao sedimento, presas a um tijolo, em profundidades semelhantes. A profundidade de incubação variou durante o experimento, conforme as características locais de variação de profundidade (120 a $60 \mathrm{~cm}$ ). Incubamos as amostras de detritos das três macrófitas e acompanhamos a perda de massa durante 60 dias, com retiradas de quatro repetições em cada tempo amostral, nos intervalos de 1, 7, 30, 45 e 60 dias após a incubação.

\section{Procedimentos Laboratoriais}

Após as retiradas, o material das bolsas foi lavado em água corrente sobre peneira de $250 \mu \mathrm{m}$ e, posteriormente, seco em estufa a $60^{\circ} \mathrm{C} / 48 \mathrm{~h}$ para obtenção da massa seca. O material seco foi triturado e realizamos análises para determinar a concentração de nitrogênio total (NT), segundo o método de Kjeldahl e de carbono orgânico, com metodologia proposta por Westlake (1965). Com esses resultados foi estabelecida a relação $\mathrm{C}: \mathrm{N}$ do detrito. Também realizamos a análise de polifenóis, segundo método adaptado de Barlocher e Graça (2005). Os invertebrados retidos na peneira foram fixados em álcool $80 \%$, triados em estereomicroscópio, identificados até o menor nível taxonômico possível e classificados em grupos tróficos funcionais (GTF) de acordo com Cummins; Merritt; Andrade (2005) e Ramírez e Gutiérrez-Fonseca (2014).

\section{Análise de Dados}

Para cada período de amostragem foram calculadas as porcentagens dos pesos remanescentes das amostras e coeficiente de degradação foliar $(\mathrm{k})$, respectivamente com as equações $\% \mathrm{R}=\left(\mathrm{M}_{\mathrm{t}} / \mathrm{M}_{0}\right) * 100$ e $\mathrm{k}=-(1 / \mathrm{t})$ * $\ln \left(\mathrm{M}_{\mathrm{t}} / \mathrm{M}_{0}\right)$ (WEBSTER; BENFIELD, 1986), onde $M_{t}$ é a massa seca em dias e $M_{0}$ é o peso inicial que foi obtido após a retirada das réplicas, que foram secas em estufa a $60^{\circ} \mathrm{C}$, até peso constante (aproximadamente 72 horas). A velocidade de decomposição foi classificada segundo os critérios de Gonçalves et al. (2014).

Calculamos abundância, riqueza de táxons e a densidade de organismos (indivíduos. g.MS- ${ }^{1}$ ) de invertebrados para cada planta em cada tempo amostral. Foi obtida a sucessão dos diferentes grupos animais e dos respectivos grupos tróficos ao longo do período de decomposição. Para avaliar diferenças entre abundâncias dos invertebrados associados aplicamos ANOVA one-way, seguida do teste post hoc de Tukey (HSD) (considerando valores significativos para $p<0,05)$ em cada planta ao longo do tempo de decomposição.

Comparamos as concentrações de nitrogênio, relação C:N e polifenóis ao longo dos 
dias de decomposição entre as espécies e entre os tempos amostrais aplicando análise de variância (ANOVA two-way) seguida do teste post hoc de Tukey (HSD), (considerando valores significativos para $p<0,05)$. As análises foram feitas utilizando-se o software Past (HAMMER; HARPER; RYAN, 2001).

\section{Resultados}

\section{Perda de massa foliar}

Nas primeiras 24 horas de experimento foi observada rápida perda de massa para as três macrófitas (Figura 1). Eichhornia crassipes teve destaque nas taxas iniciais, com massa remanescente de 23,4 $\pm 7,6 \%$. Após o período inicial, a perda de massa desta planta teve queda gradual, atingindo, no $60^{\circ}$ dia, $10,4 \pm 4,0 \%$ da massa inicial, com $\mathrm{k}=-0,348$ $\mathrm{d}^{-1}\left(\mathrm{R}^{2}=0,75\right)$. Salvinia herzogii apresentou, ao final do $60^{\circ}$ dia, $58,9 \pm 4,47 \%$ de massa remanescente, com $\mathrm{k}=-0,094 \mathrm{~d}^{-1}\left(\mathrm{R}^{2}=0,92\right)$. Já Pistia stratiotes teve sua perda de massa mais acelerada e, no $45^{\circ}$ dia, não apresentava mais detrito nas litter bags. Esta macrófita, no $30^{\circ}$ dia, apresentou $6,3 \pm 1,2 \%$ de massa remanescente, com um coeficiente de decaimento $\mathrm{k}=-0,915 \mathrm{~d}^{-1}\left(\mathrm{R}^{2}=0,98\right)$, cerca de 10 vezes mais rápido que $S$. herzogii e três vezes mais rápido que $E$. crassipes.

Figura 1 - Massa seca remanescente (\%) de Salvinia herzogii, Eichhornia crassipes e Pistia stratiotes ao longo dos dias de decomposição em uma área úmida no Sul do Brasil (barras indicam o desvio padrão da média)

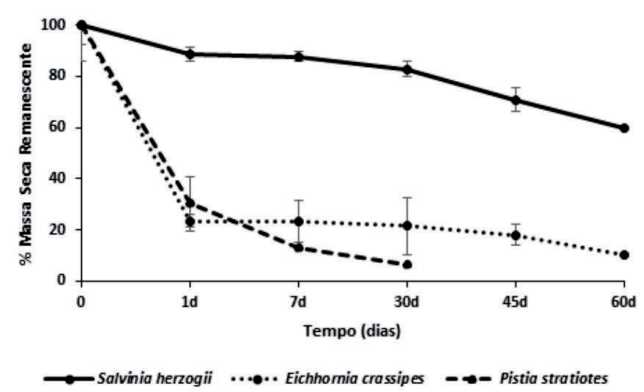

\section{Composição química do detrito}

Todas as plantas apresentaram uma diminuição na concentração de NT, nas primeiras 24 horas. Esta diminuição foi de 55\% para S. herzogii, 51\% para E. crassipes e $49 \%$ para $P$. stratiotes. A concentração inicial foi de $2,44 \%$ em massa seca para $S$. herzogii, $1,31 \%$ em E. crassipes e 2,74\% em P. stratiotes (Figura 2).

As concentrações de NT variaram ao longo do tempo e entre as plantas. Os resultados de comparação mostraram que houve diferença significativa $\left(\mathrm{F}_{4,45}=23,7 ; \mathrm{p}<0,001\right)$ nas concentrações iniciais entre $E$. crassipes e as outras duas macrófitas. Ao longo do tempo de decomposição, a concentração de NT no detrito mostrou tendência de aumento em $P$. stratiotes, atingindo $2,85 \%$ no $30^{\circ}$ dia de incubação, diferindo das outras duas plantas. Eichhornia crassipes apresentou aumento durante a decomposição, diminuindo no $60^{\circ}$ dia, com concentração de $0,96 \%$. Já $S$. herzogii, após a perda inicial, manteve a concentração relativamente constante ao longo do período de decomposição, atingindo $1,47 \%$ no $60^{\circ}$ dia (Figura 2 ).

Figura 2 - Concentração de Nitrogênio Total (\% em massa seca) ao longo do período de degradação de três macrófitas aquáticas em área úmida no Sul do Brasil (barras indicam o desvio padrão da média)

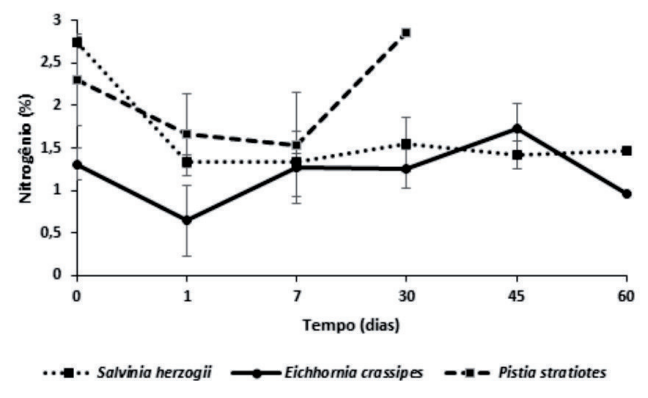

Em todas as macrófitas, a concentração de polifenóis diminuiu ao longo dos dias de 
decomposição (Figura 3). As concentrações iniciais foram maiores em $S$. herzogii, e diferiram das outras duas macrófitas $\left(\mathrm{F}_{4,45}=\right.$ $16,45 ; \mathrm{p}<0,001)$. Os resultados finais no $30^{\circ}$ dia mostraram valores significantemente menores para $P$. stratiotes, e as outras duas macrófitas apresentaram valores semelhantes até o final do experimento.

Figura 3 - Concentração de polifenóis na massa seca (UDO) ao longo do período de degradação de três macrófitas aquáticas em área úmida no Sul do Brasil (barras indicam o desvio padrão da média)

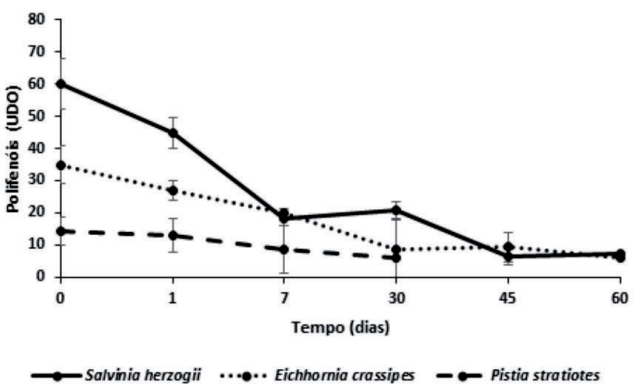

Figura 4 - Relação carbono:nitrogênio (C:N, \%) na massa seca ao longo do período de decomposição de três macrófitas aquáticas em área úmida no Sul do Brasil (barras indicam o desvio padrão da média)

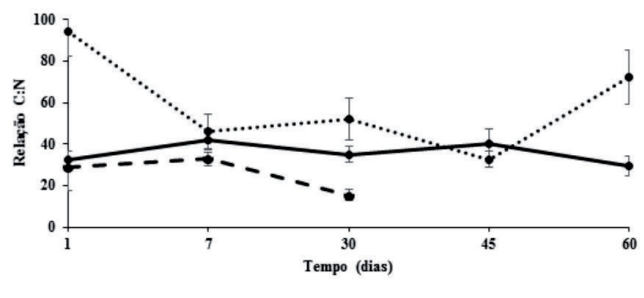

A relação C:N apresentou maiores valores iniciais em E. crassipes, diferindo das outras duas macrófitas. Os menores valores foram em $P$. stratiotes $\left(\mathrm{F}_{4,45}=12,42 ; \mathrm{p}<0,001\right)$, diferindo de E. crassipes e $S$. herzogii até o $30^{\circ}$ dia. As concentrações de $E$. crassipes e $S$. herzogii não mostraram diferença significativa nos intervalos de tempo subsequentes $\left(45^{\circ}\right.$ e $60^{\circ}$ dias). Os valores médios ao longo do período de degradação foram de 37,7 $\pm 5,35$ em $S$. herzogii, 59,42 $\pm 24,12$ em E. crassipes e 25,8 $\pm 9,23$ em $P$. stratiotes.

\section{Invertebrados colonizadores}

Foram contabilizados 1296 indivíduos distribuídos em 24 táxons, sendo 682 indivíduos identificados em $S$. herzogii $(52,6 \%$ do total), 378 indivíduos em E. crassipes $(29,2 \%)$ e 258 indivíduos em $P$. stratiotes $(19,9 \%)$. A macrófita com maior riqueza foi E. crassipes, com 20 táxons, seguida de $P$. stratiotes com 19 e $S$. herzogii com 15 . O táxon mais abundante para todas as macrófitas foi Planorbiidae, que representou $72 \%$ dos organismos em $S$. herzogii, 41,3\% em $E$. crassipes e 59,3\% em $P$. stratiotes (Tabela I). Não houve diferença significativa entre as abundâncias totais dos organismos para as três plantas estudadas $\left(\mathrm{F}_{2,67}=0,48 ; \mathrm{p}=0,61\right)$. Da mesma forma, não houve diferença nas abundâncias $\left(\mathrm{F}_{4,65}=1,014 ; \mathrm{p}=0,41\right)$ e densidade $\left(\mathrm{F}_{4,65}=0,94 ; \mathrm{p}=0,54\right)$ de invertebrados em $S$. herzogii ao longo do tempo de decomposição. Da mesma forma, em E. crassipes não houve diferença durante o tempo de decomposição em relação a abundância $\left(\mathrm{F}_{4,90}=1,56 ; \mathrm{p}=0,19\right)$ e densidade $\left(\mathrm{F}_{4,90}=1,04\right.$; $\mathrm{p}=0,27)$, assim como em $P$. stratiotes em relação a abundância $\left(\mathrm{F}_{2,51}=1,99 ; \mathrm{p}=0,15\right)$ e densidade $\left(\mathrm{F}_{2,51}=1,73 ; \mathrm{p}=0,33\right)$ dos invertebrados associados ao detrito.

A densidade de organismos para a macrófita $S$. herzogii se mostrou maior no $7^{\circ}$ dia, sendo que E. crassipes e $P$. stratiotes apresentaram a densidade maior de organismos no $1^{\circ}$ dia. Em geral, as densidades decaíram para todas as macrófitas ao longo dos dias de decomposição (Figura 5). 
Tabela I - Abundância $(\mathrm{N})$ e frequência de ocorrência (FO\%) dos invertebrados colonizadores do detrito de três macrófitas aquáticas em área úmida do Sul do Brasil (os resultados representam o total de organismos durante a decomposição)

\begin{tabular}{|c|c|c|c|c|c|c|}
\hline & \multicolumn{2}{|c|}{ Salvinia herzogii } & \multicolumn{2}{|c|}{ Eichhornia crassipes } & \multicolumn{2}{|c|}{ Pistia stratiotes } \\
\hline & $\mathrm{N}$ & FO\% & $\mathrm{N}$ & $\mathrm{FO} \%$ & $\mathrm{~N}$ & $\mathrm{FO} \%$ \\
\hline Nematoda & 1,0 & 0,1 & 8,0 & 2,1 & 3,0 & 1,2 \\
\hline Oligochaeta & 26,0 & 3,8 & 12,0 & 3,2 & 5,0 & 1,9 \\
\hline Hirudinae & 10,0 & 1,5 & 6,0 & 1,6 & 1,0 & 0,4 \\
\hline Ostracoda & 0,0 & 0,0 & 7,0 & 1,9 & 42,0 & 16,3 \\
\hline Copepoda & 23,0 & 3,4 & 52,0 & 13,8 & 13,0 & 5,0 \\
\hline Cladocera & 2,0 & 0,3 & 6,0 & 1,6 & 2,0 & 0,8 \\
\hline Amphipoda & 99,0 & 14,5 & 96,0 & 25,4 & 2,0 & 0,8 \\
\hline Bivalvia & 1,0 & 0,1 & 0,0 & 0,0 & 0,0 & 0,0 \\
\hline Ancylidae & 0,0 & 0,0 & 1,0 & 0,3 & 0,0 & 0,0 \\
\hline Physidae & 2,0 & 0,3 & 4,0 & 1,1 & 1,0 & 0,4 \\
\hline Planorbidae & 491,0 & 72,0 & 156,0 & 41,3 & 153,0 & 59,3 \\
\hline Hidracarina & 2,0 & 0,3 & 4,0 & 1,1 & 3,0 & 1,2 \\
\hline Sminthuridae & 0,0 & 0,0 & 2,0 & 0,5 & 1,0 & 0,4 \\
\hline Chironominae & 20,0 & 2,9 & 13,0 & 3,4 & 9,0 & 3,5 \\
\hline Tanypodinae & 0,0 & 0,0 & 2,0 & 0,5 & 14,0 & 5,4 \\
\hline Ceratopogonidae & 1,0 & 0,1 & 1,0 & 0,3 & 4,0 & 1,6 \\
\hline Syrphidae & 1,0 & 0,1 & 2,0 & 0,5 & 0,0 & 0,0 \\
\hline Hydrophilidae & 0,0 & 0,0 & 1,0 & 0,3 & 1,0 & 0,4 \\
\hline Hebridae & 0,0 & 0,0 & 0,0 & 0,0 & 1,0 & 0,4 \\
\hline Lutrochidae & 0,0 & 0,0 & 1,0 & 0,3 & 0,0 & 0,0 \\
\hline Elmidae & 0,0 & 0,0 & 1,0 & 0,3 & 0,0 & 0,0 \\
\hline Dytiscidae & 2,0 & 0,3 & 3,0 & 0,8 & 1,0 & 0,4 \\
\hline Caenidae & 1,0 & 0,1 & 0,0 & 0,0 & 1,0 & 0,4 \\
\hline Hydroptilidae & 0,0 & 0,0 & 0,0 & 0,0 & 1,0 & 0,4 \\
\hline Total & 682 & & 378 & & 258 & \\
\hline
\end{tabular}

Figura 5 - Densidade de invertebrados associados ao detrito das macrófitas Salvinia herzogii, Eichhornia crassipes e Pistia stratiotes em uma área úmida no Sul do Brasil

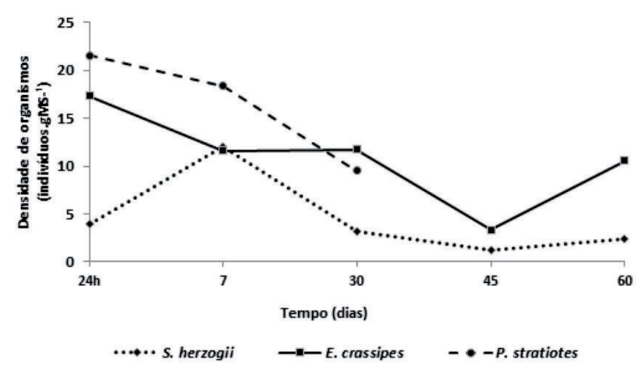

O grupo trófico funcional em maior abundância para $S$. herzogii até o $30^{\circ}$ dia foi de raspadores, seguido de coletores-catadores até o $60^{\circ}$ dia. A macrófita E. crassipes, até o $7^{\circ}$ dia, apresentou em sua maioria coletores-catadores e, posteriormente, predominância de raspadores. Já no detrito da $P$. stratiotes, até o $7^{\circ}$ dia, a colonização se deu em sua maioria por coletores-catadores e, no $30^{\circ}$ dia, por raspadores (Figuras 6, 7 e 8). 
Figura 6 - Proporção (\%) dos grupos tróficos funcionais (GTF) dos invertebrados durante a decomposição da macrófita Salvinia herzogii (Pr: predador, Co-Fi: coletor-filtrador, Co-Ca: coletor-catador, Ra: raspador, Fr: fragmentador)

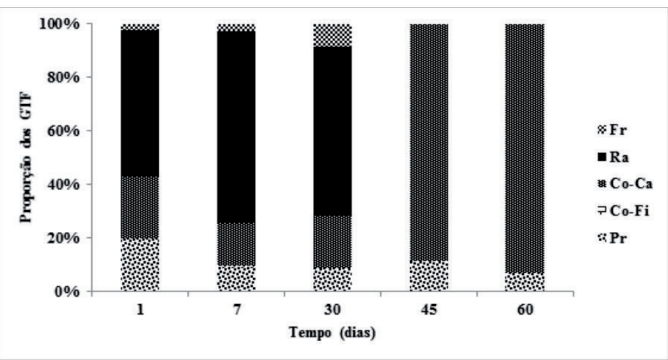

Figura 7 - Proporção (\%) dos grupos tróficos funcionais (GTF) dos invertebrados durante a decomposição da macrófita Eichhornia crassipes (Pr: predador, Co-Ca: coletor-catador, Ra: raspador, Fr: fragmentador)

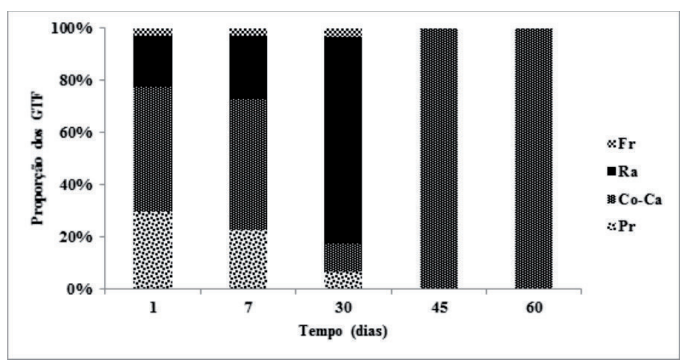

Figura 8 - Proporção (\%) dos grupos tróficos funcionais (GTF) dos invertebrados durante a decomposição da macrófita Pistia stratiotes (Pr: predador, Co-Ca: coletor-catador, Ra: raspador, Fr: fragmentador)

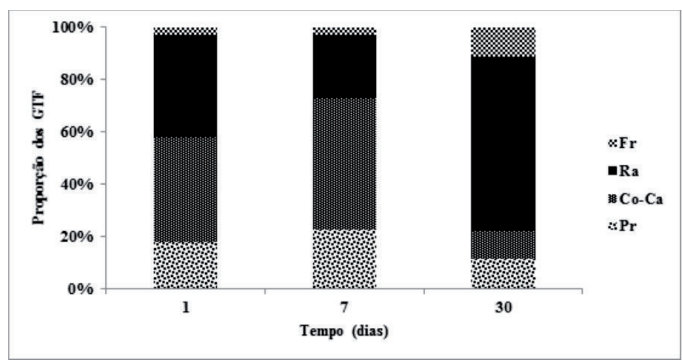

\section{Discussão}

Embora com diferenças em relação à velocidade de degradação, todas as três plantas foram classificadas como de decomposição rápida, com $\mathrm{k}>-0,0173 \mathrm{~d}^{-1}$, segundo critérios propostos por Gonçalves et al. (2014). Os coeficientes de decomposição encontrados em nosso estudo estão acima daqueles repor- tados na literatura para essas espécies. Por exemplo, P. stratiotes apresentou velocidade cerca de 15 vezes maior e $E$. crassipes cerca de 18 vezes mais rápida do que a reportada por Chimney e Pietro (2006) em áreas úmidas na Flórida $\left(\mathrm{k}=-0,0508 \mathrm{~d}^{-1} \mathrm{e}-0,0191 \mathrm{~d}^{-1}\right.$ respectivamente). Coeficiente de decomposição de $-0,0220 \mathrm{~d}^{-1}$ é citado para $E$. crassipes por Poi de Neiff et al. (2006) em planície de inundação do rio Paraná, valor aproximadamente 15 vezes mais lento que nosso resultado.

Vários fatores influenciam a velocidade de decomposição de detritos em ecossistemas aquáticos, sendo os mais reportados na literatura variáveis limnológicas como temperatura, $\mathrm{pH}$, profundidade e estado trófico do ambiente (WEBSTER; BENFIELD, 1986). Além das variáveis ambientais, a composição química do detrito, particularmente altas concentrações de nitrogênio e baixa relação carbono:nitrogênio (C:N) aumentam a palatabilidade, influenciando de maneira efetiva a velocidade desse processo (LIGEIRO et al., 2010; TONELLO et al., 2014). Nossos resultados confirmaram a literatura citada, mostrando que a macrófita com menor relação C:N - P. stratiotes - foi a que apresentou maior velocidade de decomposição, não sendo encontrada massa remanescente a partir do $45^{\circ}$ dia do experimento.

Por outro lado, altas concentrações de compostos fenólicos em vegetais, principalmente taninos, têm, reconhecidamente, a função de inibir herbívoros, tornando os vegetais impalatáveis aos fitófagos, inibindo a ação das enzimas digestivas e o consumo de folhas, devido a sua toxicidade (MONTEIRO; ALLBUQUERQUE; ARAÚJO; 2005). É citado que polifenóis são eficientes antimicrobianos, reduzindo o crescimento de fungos e bactérias (SCALBERT, 1991). Tais características fazem com que detritos com alta concentração desses compostos tenham tempo de decomposição aumentado, tanto por dificultar a ação dos invertebrados 
fragmentadores (GRAÇA, 2001) como, provavelmente, também pelo retardo no condicionamento microbiano (RAHMAN et al., 2013). Nossos resultados confirmaram nossa primeira hipótese, pois a macrófita com maiores concentrações de nitrogênio e menores concentrações de polifenóis $-P$. stratiotes - apresentou a maior velocidade de decomposição.

Ao longo da decomposição, o aumento na concentração de nutrientes é atribuído ao condicionamento microbiano que ocorre ao longo do processo (KELLEY; JACK, 2002; TELÖKEN et al., 2014), com aumento da biomassa fúngica e bacteriana e consequente enriquecimento do detrito com nitrogênio. Os menores valores da relação $\mathrm{C}: \mathrm{N}$ em $P$. stratiotes indicaram que essa macrófita possui um detrito com maior qualidade nutricional entre as três estudadas. No entanto, essa melhor qualidade nutricional não se refletiu em maior abundância de invertebrados, visto que $P$. stratiotes foi a macrófita com menor abundância de organismos, embora sem diferença significativa em relação às outras duas plantas. Em outros estudos com decomposição de macrófitas em áreas úmidas, densidades de invertebrados costumam ser maiores em plantas de perda de massa mais rápida (WEBSTER; BENFIELD, 1996; POI DE NEIF; GALASSI; FRANCESCHINI, 2009). $P$. stratiotes apresentou a maior densidade no primeiro dia de decomposição, o que pode ser explicado pela melhor qualidade nutricional, mas sua rápida perda de massa talvez tenha sido responsável pela diminuição do número de invertebrados ao longo da decomposição.

Existem dois aspectos a serem considerados em relação aos organismos colonizadores do detrito das macrófitas estudadas. O primeiro refere-se ao fator tempo, pois macrófitas com maior tempo de degradação podem favorecer a permanência de uma comunidade associada ao detrito com maior estruturação (LIGEIRO et al., 2010). O segundo refere-se à utilização dos detritos predominantemente como substrato e refúgio, e não como recurso alimentar. Nesse aspecto, várias pesquisas demonstraram a baixa densidade e virtual ausência de fragmentadores em sistemas tropicais e subtropicais (GONÇALVES; GRAÇA; CALLISTO, 2007; TELÖKEN et al., 2014; ALBERTONI et al., 2018). No entanto, um grupo trófico específico tem se mostrado abundante nesses sistemas - os raspadores. Organismos raspadores podem incrementar a atividade microbiana no detrito, tanto aumentando a aeração do mesmo (MASON; BRYANT, 1975) quanto enriquecendo o detrito com nutrientes por meio de suas fezes (POLUNIN, 1982). Além disso, esses organismos podem atuar como fragmentadores, no sentido de facilitar a quebra do detrito pela sua atividade alimentar, proporcionando maior velocidade para o condicionamento microbiano (RAMÍREZ; GUTIERRES- FONSECA, 2014). Gastropoda são um bom exemplo, com a utilização da rádula removendo também tecido do detrito pela atividade de sua alimentação (RAMÍREZ; GUTIÉRRES-FONSECA, 2014). A presença de Chironomidae em grande número em detritos também pode se mostrar como catalisador do processo de decomposição, conforme colocado por Biasi et al. (2013). Alto número de gastrópodes planorbídeos foram registrados por Varga (2003) na decomposição de Phragmites australis, e o autor atribuiu a presença desses organismos a uma maior velocidade de decomposição de rizomas dessa macrófita.

Nossos resultados mostraram que nas três macrófitas foi observada presença muito pequena de organismos fragmentadores, de acordo com a classificação da literatura corrente. No entanto, o grupo predominante nas três plantas foi de planorbídeos, moluscos raspadores, e sugerimos que esses organismos podem ter auxiliado na velocidade de decomposição, tanto como facilitadores para 
o condicionamento microbiano, quanto pela ação mecânica de fragmentação dos detritos. Essa influência de raspadores provavelmente foi a de maior importância para aumento da velocidade de perda de massa dos detritos nas macrófitas estudadas, tendo em vista que Chironomidae, principalmente Chironominae, foi o segundo grupo em importância na macrófita $P$. stratiotes, mais rápida, não apresentando ocorrência predominante nas outras duas plantas estudadas. Essas características de estruturação da comunidade de invertebrados, associadas ao menor teor de polifenóis e menor relação $\mathrm{C}: \mathrm{N}$, foram, provavelmente, os fatores responsáveis pela rápida degradação de $P$. stratiotes.

Assim, os resultados desta pesquisa mostraram que, das três hipóteses que direcionaram o desenvolvimento do estudo, foi confirmado que a macrófita com maior concentração de nitrogênio e menor teor de polifenóis apresentou a perda de massa mais rápida ( $P$. stratiotes). Já a maior densidade de organismos foi encontrada na planta que apresentou velocidade intermediária de decomposição, em relação às outras duas (E. crassipes); e a maior abundância total de organismos foi verificada na planta com menor velocidade (S. herzogii), concordando com as observações de Ligeiro et al. (2010). Desta forma, nossa segunda hipótese foi parcialmente comprovada. Tínhamos também a premissa de encontrar baixo número de organismos fragmentadores strictu sensu, o que efetivamente se confirmou.
No entanto, a grande presença de organismos raspadores, representado cerca de $50 \%$ do total de organismos em todas as macrófitas, faz supor que o papel de fragmentação de detritos por invertebrados em áreas úmidas subtropicais esteja sendo subestimado. Provavelmente, o papel de fragmentação não esteja relacionado com a utilização do detrito como recurso alimentar, mas seja realizado principalmente por comportamento alimentar de alguns organismos, auxiliando a transformação de MOPG dos detritos em MOPF, assim disponibilizando recurso alimentar a outros grupos, como coletores. Além disso, a alta velocidade de perda de massa dos três detritos avaliados faz supor que a atividade dos raspadores pode, efetivamente, facilitar o condicionamento microbiano, consistindo em um efeito sinérgico na velocidade de decomposição do detrito de macrófitas aquáticas em áreas úmidas subtropicais.

Desta forma, nossos resultados reforçam as conclusões apresentadas por Ligeiro et al. (2010), Biasi et al. (2013) e Ramírez; Gutiérres-Fonseca (2014) em relação ao papel de diferentes organismos na fragmentação e velocidade de perda de massa de diferentes detritos nos ecossistemas aquáticos. Por outro lado, os resultados de alto coeficiente de decomposição das macrófitas estudadas reforça a necessidade de pesquisas experimentais para determinar o real papel dos invertebrados na degradação de detritos em sistemas de áreas úmidas subtropicais. 


\section{REFERÊNCIAS}

ABELHO, M. From litterfall to breakdown in streams: a review. The Scientific World Journal, v. 1, p. 656-680, 2001.

ALBERTONI, E. F.; PRELLVITZ, L. J.; PALMA-SILVA, C. Macroinvertebrate fauna associated with Pistia stratiotes and Nymphoides indica in subtropical lakes (south Brazil). Brazilian Journal of Biology, v. 67, n.3, p. 499-507, 2007.

ALBERTONI, E. F.; HEPP, L. U.; CARVALHO, C.; PALMA-SILVA, C. Invertebrate composition in submerged macrophyte debris: habitat and degradation time effects. Ecologia Austral, v. 28, p. 93-103, 2018.

ASAEDA, T.; TRUNG, V. K.; MANATUNGE, J. Modeling the effects of macrophyte growth and decomposition on the nutrient budget in Shallow Lakes. Aquatic Botany, v. 68, p. 217-237, 2000.

BÄRLOCHER, F.; GRAÇA, M.A.S. Total phenolics. In: GRAÇA M.A.S.; BÄRLOCHER, F; GESSNER, M.O (eds.). Methods to Study Litter Decomposition: A Practical Guide. London, Springer, 2005, p. 97-100.

BIASI, C.; TONIN, A. M.; RESTELLO, R. M.; HEPP, L. U. The colonisation of leaf litter by Chironomidae (Diptera): The influence of chemical quality and exposure duration in a subtropical stream. Limnologica, v. 44, p. 427-433, 2013.

CARVALHO, C., HEPP, L.U. ALBERTONI, E. F.; PALMA-SILVA, C. Decomposition of macrophytes in a shallow subtropical lake. Limnologica, v. 53, p. 1-9, 2015.

CHIMNEY, M. J.; PIETRO, K. C. Decomposition of macrophyte litter in a subtropical constructed wetland in south Florida (USA). Ecological Engineering, v. 27, p. 301-32, 2006.

CUMMINS, K. W.; MERRITT, R. W.; ANDRADE, P. C. N. The use of invertebrate functional groups to characterize ecosystem attributes in selected streams and rivers in south Brazil. Studies in Neotropical Fauna and Environment, v. 40, p. 69-89, 2005.

DUDGEON, D.; WU, K. K. Y. Leaf litter in a tropical stream: food or substrate for macroinvertebrates? Archives für Hydrobiologie, v. 146, p.65-82, 1999.

GESSNER, M. O.; CHAUVET, E.; DOBSON, M. A Perspective on leaf litter breakdown in stream. Oikos, v. 85, p. 377-384, 1999.

GONÇALVES, J. F.; GRAÇA, M. A. S.; CALLISTO, M. Leaf-litter breakdown in 3 streams in temperate, mediterranean, and tropical Cerrado climates. Journal of North American Benthological Society, v. 25, n. 2, p. 344-355, 2006.

GONÇALVES, J.F.; GRAÇA, M.; CALLISTO, M. Litter decomposition in a Cerrado savannah stream is retarded by leaf toughness, low dissolved nutrients and a low density of shredders.

Freshwater Biology, v. 52, p. 1440-1451, 2007.

GONÇALVES, J.F.; MARTINS, R.T.; OTTONI, B.M.T.; COUCEIRO, S.E.M. Uma visão sobre a decomposição foliar em sistemas aquáticos Brasileiros. In: Insetos aquáticos na Amazônia brasileira: taxonomia, biologia e ecologia HAMADA, N.; NESSIMIAN, J. L.; QUERINO. R. B. Manaus: Editora do INPA, 6. ed. 2014, p.79-116.

GRAÇA, M. A. S. The role of invertebrates on leaf litter decomposition in streams - a review.

International Review of Hydrobiology, v. 86, p. 383-393, 2001. 
HAMMER, Ø.; HARPER, D.A.T.; RYAN, P. D. PAST: Paleontological Statistics Software Package for Education and Data Analysis. Palaeontologia Electronica, v. 4, n.1, 9p, 2001.

HEPP, L. U.; DELANORA, R.; TREVISAN, A. Compostos secundários durante a decomposição foliar de espécies arbóreas em um riacho do Sul do Brasil. Acta Botanica Brasilica, v. 2, n. 2, p. 407-413, 2009.

KELLEY, R. H.; JACK, J. D. Leaf litter decomposition in an ephemeral karst lake (Chaney Lake, Kentucky, U.S.A.). Hydrobiologia, v. 482, p. 41-47, 2002.

LIGEIRO, R.; MORETTI, M. S.; GONÇALVES, J. F., CALLISTO, M. What is more important for invertebrate colonization in a stream with low-quality litter inputs: exposure time or leaf species? Hydrobiologia, v. 654, n. 1, p. 125-136, 2010.

MASON, C. F.; BRYANT, R. J. Production, Nutrient Content and Decomposition of Phragmites Communis Trin. and Typha angustifolia L. Journal of Ecology, v. 63, n. 1, p. 71-95, 1975.

MONTEIRO, J.M.; ALLBUQUERQUE, U.P.; ARAÚJO, E.L. Taninos: uma abordagem da química à ecologia. Química Nova, v. 28, p. 892-896. 2005.

MORETTI, M.; GONÇALVES, J. F.; CALLISTO, M. Leaf breakdown in two tropical streams: Differences between single and mixed species packs. Limnologica, v. 37, p. 250-258, 2007.

NEGRISOLI, E.; CORREAA, M. R.; VELINI, E. D.; BRAVIN, L. F.; MARCHI, S. R.;

CAVENAGHI, A. L.; ROSSI, C. V. S. Estudo da degradação da biomassa de três espécies de plantas aquáticas no reservatório da UHE de Americana-SP. Planta daninha, v. 24, n. 2, p. 221-227, 2006.

PALMA-SILVA, C.; ALBERTONI, E. F.; TRINDADE, C.; PEREIRA, S.; FURLANETTO, L.; SILVA, C. Caracterização dos ecossistemas aquáticos associados à Planície Costeira Sul do Rio Grande do Sul. In: MARTINS, S.; ALBERTONI, E. F.; GONÇALVES, C.; COLARES, I. (Org.), Ambientes aquáticos do Rio Grande do Sul: propostas alternativas para o ensino na educação básica. Editora da Furg, Rio Grande, 2012. p. 22-54.

POI DE NEIFF, A.; NEIFF J. J.; CASCO, S. L. Leaf litter decomposition in three wetland types of the Parana River floodplain. Wetlands, v. 26, p. 558-66, 2006.

POI DE NEIFF, A.; GALASSI, M. E.; FRANCESCHINI, M. C. Invertebrate assemblages associated with leaf litter in three floodplain wetlands of the Parana river. Wetlands, v. 29, n.3, p. 896-906, 2009.

POLUNIN, N. V. C. Effects of the freshwater gastropod Planorbis carinatus on reed (Phragmites australis) litter microbial activity in an experimental system. Freshwater Biology, v. 12, n. 6, p. 547-552, 1982.

RAHMAN, M. M.; TSUKAMOTO, J.; TOKUMOTO, Y.; SHUVO, A. R. Md. The Role of Quantitative Traits of Leaf Litter on Decomposition and Nutrient Cycling of the Forest Ecosystems. Journal of Forest Science, v. 29, n. 1, p. 38-48, 2013.

RAMÍREZ, A.; GUTIÉRREZ-FONSECA, P. E. Functional feeding groups of aquatic insect families in Latin America: a critical analysis and review of existing literature. Revista de Biologia Tropical, v. 62 (Suppl. 2), p. 155-167, 2014.

ROLON, A. S.; MALTCHIK, L. Environmental factors as predictors of aquatic macrophyte richness and composition in wetlands of southern Brazil. Hydrobiologia, v. 556, n. 1, p. 221-231, 2006.

SCALBERT, A. Antimicrobial properties of tannins. Phytochemistry, v. 30, n.12, p. 3875-3883, 1991.

TELÖKEN, F.; ALBERTONI, E.F.; PALMA-SILVA, C. Leaf degradation of Salix humboldtiana Willd. (Salicaceae) and invertebrate colonization in a subtropical lake (Brazil). Acta Limnologica Brasiliensis, v. 23, n. 1, p. 30-41, 2011. 
TELÖKEN, F.; ALBERTONI, E. F.; HEPP, L. U.; PALMA-SILVA, C. Invertebrados aquáticos associados a serapilheira de Salix humboldtiana em um riacho subtropical. Ecología Austral, v. 24, p. 220-228, 2014.

TONELLO, G.; LOUREIRO, R. C.; KRAUSE, P.; SILVA, C.S.; ONGARATTO, R. M.; SEPP, S.; RESTELLO, R. M.; HEPP, L.U. Colonização de invertebrados durante a decomposição de diferentes detritos vegetais em um riacho subtropical. Revista Brasileira de Biociências, v. 12, n. 2, p. 98-105, 2014.

TRINDADE, C. R. T.; PEREIRA, S. A.; ALBERTONI, E. F.; PALMA-SILVA, C. Caracterização e importância das macrófitas aquáticas com ênfase nos ambientes límnicos do Campus Carreiros FURG, Rio Grande, RS. Cadernos de Ecologia Aquática, v. 5, p. 1-22. 2010.

VARGA, I., Structure and changes of macroinvertebrate community colonizing decomposing rhizome litter of common reed at Lake Fert"o/Neusiedler See (Hungary). Hydrobiologia, v. 506-509, p. 413-420, 2003.

WALLACE, J. B.; WEBSTER. J. R. The role of macroinvertebrates in stream ecosystem function. Annual Review of Entomology, n. 41, p.115-139, 1996.

WEBSTER, J.R.; BENFIELD, E. F. Vascular plant breakdown in freshwater ecosystems. Annual review of Ecology Evolution and Systematics, v. 17, p. 567-594, 1986.

WESTLAKE, D. F. Some basic data for investigations of the productivity of aquatic macrophytes. Memorie dell'Istituto Italiano di Idrobiologia, v. 18, p. 229-248, 1965. 
